\title{
An Effective Approach to Job Scheduling in Decentralized Grid Environment
}

\author{
G.K.Kamalam \\ Senior Lecturer/CSE \\ Kongu Engineering College \\ Perundurai, Erode
}

\author{
V.Murali Bhaskaran \\ Principal \\ Paavai College of Engineering \\ Pachal, Namakkal
}

\begin{abstract}
Scheduling of jobs and resource management are the important challenging work in a grid environment. Processing time minimization of the jobs arriving at any computer site in a grid system is one of the major objectives in the research area of computing. In this paper, we propose a decentralized grid system model as a collection of clusters. We then introduce a decentralized job scheduling algorithms which performs intra cluster and inter cluster (grid) job scheduling. In this paper, we apply Divisible Load Theory (DLT) and Least Cost Method (LCM) to model the grid scheduling problem involving multiple resources within an intra cluster and inter cluster grid environment. The decentralized job scheduling algorithm is proposed for both reducing processing time and processing cost. The proposed decentralized hybrid job scheduling algorithm employs the DLT and LCM technique and decentralized divisible job scheduling algorithm employs DLT technique and produces reduced processing time and reduced processing cost. The result shows that the gap between the decentralized job scheduling algorithm and centralized job scheduling algorithm is widening as the number of jobs is increased.
\end{abstract}

\section{Keywords}

Job Scheduling, Heuristic Algorithm, Load Balancing, Cluster, Coordinator Node, Worker Node.

\section{INTRODUCTION}

A computational grid is emerging as a wide-scale distributed computing infrastructure where user jobs can be executed on either intra cluster or inter cluster computer systems [7,9]. Computational grids have the ability for solving large-scale scientific problems using heterogeneous and geographically distributed resources dynamically at run time depending on their availability, capability, performance, cost and quality of service requirements [6,8]. Resource management and job scheduling in grid environment based on clusters is one of the challenging task [5]. Scheduling is an important problem in computational grid [14]. The grid environment is dynamic in nature, in other words, the number of resources and jobs to be scheduled are usually variable. This kind of feature of grid makes the scheduling problem a complex optimization problem [15]. The effective utilization of grid is the efficient scheduling of jobs to the available resources.

Many heuristic algorithms have been designed to solve the job scheduling problems. Based on the time at which the scheduling decisions are made, these algorithms are divided into static and dynamic scheduling [10]. In the static mode, a firm estimate of the completion time is made in advance of the actual execution. The advantage of the static model is that it is easier to program from the scheduler's point of view. The allocation of job to resource is fixed a priori, and estimating the cost of job is also easy. The disadvantage is that the cost estimate based on the static information is not accurate because in some situations, the resource selected for executing a job fails due to network failure, or is so heavily loaded with jobs that its response time is more than expected. Dynamic scheduling is applied when the jobs are arrived dynamically. It is used when it is difficult to estimate the computation time of a job [13].

The primary importance is to design an efficient scheduling algorithm for minimizing the total processing time of the jobs. This is achieved by distributing the jobs among the resources available in the grid system. The aim is to minimize the overall processing time and the overall processing cost using specified decentralized job scheduling algorithm. This scheduling technique is used to effectively schedule jobs to the available resources to reduce the processing time and processing cost of the jobs. This research is concerned with scheduling of jobs in distributed systems with divisible jobs.

The aim of this paper is to present a decentralized hybrid job scheduling algorithm and decentralized divisible job scheduling algorithm adapted to heterogeneous grid computing environment. In this paper, we compare two classes of scheduling algorithms centralized and decentralized job scheduling algorithms. We model the grid as a group of clusters. Group of users submit jobs to the various clusters. In centralized scheduling, the scheduler of each cluster is responsible for scheduling of submitted jobs. In decentralized scheduling, jobs though submitted locally can be migrated to another cluster in order to reduce the processing time of the jobs.

The rest of the paper is organized as follows: Section 2 summarizes the related work. Section 3 discusses the decentralized grid architecture model. Section 4 describes the design of decentralized job scheduling algorithm. Section 5 compares the decentralized and centralized job scheduling algorithms. Section 6 concludes the paper.

\section{RELATED WORK}

Divisible load theory is designed to solve the challenging problem of allocating the independent jobs to the available resources on a grid $[2,3,12]$. A divisible job is one that can be arbitrarily divided into independent sub jobs. These sub jobs can be executed in any order on platforms ranging from one single cluster to large distributed grid systems [11]. The processing time of each sub job is small compared to the time to process the whole job.

In LCM method, the jobs are allocated to the resource with the least allocation cost [1]. 
In [1] introduced a decentralized load balancing algorithm, that considers load index as a decision parameter for scheduling of jobs within intra cluster and inter cluster grid environment.

\section{DECENTRALIZED GRID ARCHITECTURE MODEL}

We have proposed a decentralized grid scheduling architecture model for scheduling jobs in grid consists of a collection of clusters where cluster server are treated as Coordinator Nodes $(\mathrm{CN})$. Each cluster consists of more number of Worker Nodes (WN). Each worker node has different processing powers. The worker nodes within the cluster are interconnected through a high speed local area network. The clusters are connected through a wide area network.

The jobs generated by the users are submitted to the coordinator nodes. A scheduler then partitions the jobs into sub jobs and schedules. Dispatcher then dispatches the partitioned sub jobs to the appropriate resources in the intra cluster or inter cluster grid environment. Grid Information Centre (GIC) maintains the memory and CPU utilization value along with the registration information of $\mathrm{CN}$.

\section{DECENTRALIZED JOB}

\section{SCHEDULING ALGORITHMS (DJSA)}

\subsection{Decentralized Makespan Local Job Scheduling Algorithm (DMLJSA)}

The grid system consists of a cluster with a Coordinator Node denote $\mathrm{CN}$, connected to a set of $\mathrm{n}$ Worker Node denoted as $\mathrm{WN}_{1}, \mathrm{WN}_{2}, \ldots, \mathrm{WN}_{\mathrm{n}}$. Each user is assigned to a particular cluster. The jobs submitted by the user will be executed in the originating cluster or it will be migrated to any of the clusters.

Let $\mathrm{k}$ be the number of jobs and $\mathrm{n}$ be the number of worker nodes in each cluster. Let 1 represent the number of users and $\mathrm{m}$ represents the number of clusters in a grid system. More formally,



Set of clusters, $C=\left\{\mathrm{Cl}_{w} \mathrm{C2}_{v} \mathrm{C} 3_{w} \mathrm{Cm}\right\}$

Set of users, $U=\left\{U 1_{w} U 2_{2} U 3_{w a x} U 1\right\}$

Set of workernodes,



where

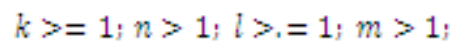

$\mathrm{WN}_{\mathrm{nm}}$ is the $\mathrm{m}^{\text {th }}$ worker node of $\mathrm{n}^{\text {th }}$ cluster. grid system

$\mathrm{J}_{\mathrm{k}}$ is the $\mathrm{k}^{\text {th }}$ job of the set of jobs submitted to the $\mathrm{n}^{\text {th }}$ cluster.

$\mathrm{PC}_{\mathrm{nm}}$ is the processing cost of $\mathrm{m}^{\text {th }}$ worker node of cluster.

$$
\mathrm{CT}_{\mathrm{ij}} \text { is the completion time of } \mathrm{j}^{\text {th }} \text { worker node of } \mathrm{i}^{\text {th }}
$$

When the job enters cluster, the scheduler receives the information from GIC and finds the worker node from the entire cluster whose completion time is minimum and assigns the job to that particular worker node.
Decentralized makespan local job scheduling algorithm is as follows:

Step1: The completion time information of each WN of each cluster is updated at GIC or CN of a cluster periodically.

Step2: If the job set $\mathbf{J}$ is empty then go to step6.

Step3: If a new job arrives at $\mathrm{CN}$ of a cluster $\mathrm{C}_{\mathrm{i}}$, the scheduler receives the completion time information of a worker node of each cluster from GIC and assigns the job to the worker node with minimum processing time. The processing time is calculated as follows:

$$
\text { makespan }=\text { clusterlist }[0] \text {. WNList }[0] \text {.uptime }
$$

for each cluster $\mathrm{Ci}_{x} 1 \leq i \leq$ noof clusters

for each worker node $W_{N j} 1 \leq j \leq$ workernodes

if (clusterlist[i], WNList[j].uptime > makespan)

makespan $=$ clusterlist [i]. WNList [j].uptime

Step4: Repeat step 4 until all jobs is scheduled.

Step5: The processing cost is calculated as:

$$
\begin{aligned}
& \text { for } i=1 \text { to } m \\
& \text { for } j=1 \text { to } n \\
& \text { Total cost = Total cost }+ \text { Crij } \& \text { PCij }
\end{aligned}
$$

Step6: END.

\subsection{Decentralized Cost Local Job Scheduling Algorithm (DCLJSA)}

When the job enters cluster, the scheduler receives the information from GIC and finds the worker node from the entire cluster whose completion time and processing cost is minimum and assigns the job to that particular worker node.

Decentralized cost local job scheduling algorithm is as follows:

Step1: The completion time information of each WN of each cluster is updated at GIC or $\mathrm{CN}$ of a cluster periodically.

Step2: If the job set J is empty then go to step6.

Step3: If a new job arrives at $\mathrm{CN}$ of a cluster $\mathrm{C}_{\mathrm{i}}$, the scheduler receives the completion time information of a worker node of each cluster from GIC and assigns the job to the worker node with minimum processing time and minimum processing cost. The processing time is calculated as follows:

makespan $=$ clusterlist $[0]$, WNList $[0]$,uptime

for each cluster $\mathrm{Ci}_{v} 1 \leq i \leq$ noof clusters

for each worker node WN $j, 1 \leq j \leq$ workernodes

if (clusterlist[i], WNList[j].uptime > makespan)

makespan $=$ clusterlist [i]. WNList [j].uptime

Step4: Repeat step 4 until all jobs is scheduled. 
Step5: The processing cost is calculated as:

$$
\begin{aligned}
& \text { for } i=1 \text { to } m \\
& \text { for } j=1 \text { to } n \\
& \text { Total cost = Total cost }+ \text { Crij } \& \text { PCij }
\end{aligned}
$$

Step6: END.

\subsection{Decentralized Divisible Job Scheduling Algorithm (DDJSA)}

A divisible job $\mathrm{J}_{\mathrm{i}}$ is divided into jobs of equal size.

Let $\mathrm{k}$ be the number of jobs and $\mathrm{q}$ is the number of partitions of a job.

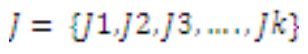

where $\mathrm{J}$ is a set of $\mathrm{k}$ jobs and $\mathrm{k}>=1$

Each job is split into q sub jobs

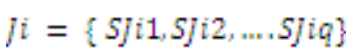

where $\mathrm{q}>=1$

Decentralized Divisible job scheduling algorithm is:

Step1: The completion time of WN of every cluster is maintained at GIC or $\mathrm{CN}$ of cluster periodically.

Step2: If $\mathbf{J}$ is empty, go to step 8 .

Step3: If a job arrives, divide the job into sub jobs to the maximum of five partitions.

Step4: Find the worker node with minimum processing time from all the clusters as follows:

makespan $=$ clusterlist $[0]$, WNList $[0]$.uptime

for each cluster $C_{v} 1 \leq i \leq$ noof clusters

for each worker node $W N_{j}, 1 \leq j \leq$ workernodes

if (clusterlist[i], WNList[j].uptime > makespan)

makespan $=$ clusterlist $[i] \cdot$ WNList [i]. uptime

Step5: Repeat step 4 until all the ' $q$ ' sub jobs are processed.

Step6: Repeat step 3 to 4 until all the jobs are scheduled.

Step7: Calculate the processing cost.

$$
\begin{aligned}
& \text { for } i=1 \text { to } m \\
& \qquad \text { for } j=1 \text { to } \mathrm{n} \\
& \quad \text { Total cost = Total cost }+ \text { Crij } \& \text { PCij }
\end{aligned}
$$

Step 8: END.

\subsection{Decentralized Hybrid Job Scheduling Algorithm (DHJSA)}

In static scheduling the scheduler needs to know the execution time of every job in advance. If this information is not accurate the scheduling decisions may be inefficient. In real time systems, the estimation of job execution time is a hard problem. To overcome this inefficiency, we propose the decentralized hybrid job scheduling algorithm. First, the users are assigned to the various clusters. Jobs that are mutually independent are submitted to the different $\mathrm{CN}$ of various clusters. The scheduler than partitions the job into sub jobs to the maximum of five partitions and are placed in a global job set $\mathrm{J}$. The $\mathrm{CN}$ receives the completion time information of each worker node in each cluster and communicates this information to the GIC periodically, which is later on used for the allocation of sub jobs to the worker node among clusters. Upon receiving this information from GIC, the scheduler then schedules the sub jobs to the worker node of any cluster whose processing time * processing cost is minimum.

Decentralized hybrid job scheduling algorithm is described as:

Step1: If there is any completion time information from $\mathrm{CN}$ or WN then update the information at GIC or $\mathrm{CN}$.

Step2: If $\mathbf{J}$ is empty then go to step 10.

Step 3: If a job $\mathrm{J}_{\mathrm{i}}$ completes the execution of all its sub jobs and was migrated to another cluster then dispatch this job along with results to the generated cluster and remove the job from the job set $\mathrm{J}$.

Step 4: If a new job arrives at $\mathrm{CN}$ of any cluster $\mathrm{C}_{\mathrm{i}}$ then partition the job into maximum of 5 equal partitions and then add it to the job set.

Step 5: Among all the clusters find the worker node with minimum processing time and allocation cost. The processing time

makespan $=$ clusterlist $[0]$. WNList $[0]$.uptime

for each cluster $C_{v} 1 \leq i \leq$ noof clusters

for each worker node WNj, $1 \leq j \leq$ workernodes

if (clusterlist[i]. WNList[j].uptime > makespan)

makespan $=$ clusterlist [i]. WNList [j].uptime

Step6: $\mathrm{CN}$ at cluster $\mathrm{C}_{\mathrm{i}}$ then dispatches the sub job to the worker node $\mathrm{WN}_{\min }$

Step7: Repeat step 5 to 6 until all sub jobs is scheduled.

Step8: Repeat step 4 to 6 until the job set is empty.

Step9: Calculate the processing cost.

$$
\begin{aligned}
& \text { for } i=1 \text { tom } \\
& \qquad \text { for } j=1 \text { ton } \\
& \text { Total cost = Total cost }+ \text { CTij } \& \text { PCij }
\end{aligned}
$$

Step10: END.

\section{COMPUTATIONAL RESULTS}

In this section, the performance of decentralized job scheduling algorithm is studied based on the simulation parameters of [4] and are shown in Table 1.

Table 1. Simulation Parameters

\begin{tabular}{|l|l|}
\hline Parameters & Value \\
\hline No. of Clusters & 10 \\
\hline No. of worker nodes per cluster & 10 \\
\hline Processing power of worker & $500-5000$ MIPS \\
\hline Job length & $2,50,000-$ \\
& $6,50,000 \mathrm{MI}$ \\
\hline Cost & $1-5 \mathrm{G} \$$ unit \\
\hline No. of users & 5 \\
\hline No. of Jobs & $50-500$ \\
\hline
\end{tabular}


The DJSA performance measurement is measured based on the three parameters: Total processing time, Total cost and Number of jobs. The result of DJSA and without DJSA (WDJSA) shows that the DJSA is more efficient. The total processing time of completing the jobs of DDJSA algorithm is minimum compared to that of the WDJSA algorithm is shown in Table 2 and Table 3 respectively. The total processing cost of completing the jobs of DHJSA algorithm is minimum compared to that of the WDJSA algorithm is shown in Table 4 and Table 5 respectively. Figure 2 and Figure 3 shows the impact of DJSA and WDJSA on the total processing time and the total cost of completing the jobs.

Table 2. Total Processing Time of WDJSA Algorithm

\begin{tabular}{|c|c|c|c|c|}
\hline $\begin{array}{c}\text { No. of } \\
\text { Jobs }\end{array}$ & WDCLJSA & WDMLJSA & WDHJSA & WDDJSA \\
\hline 50 & 907 & 502 & 486 & 313 \\
\hline 100 & 2811 & 1508 & 2333 & 1102 \\
\hline 150 & 4644 & 1829 & 2816 & 1116 \\
\hline 200 & 5863 & 2546 & 3037 & 1324 \\
\hline 250 & 6300 & 3340 & 3973 & 2114 \\
\hline 300 & 7362 & 3432 & 3651 & 1710 \\
\hline 350 & 10695 & 4581 & 7173 & 3083 \\
\hline 400 & 16615 & 6168 & 10272 & 3844 \\
\hline 450 & 5665 & 4519 & 3578 & 2866 \\
\hline 500 & 15177 & 5618 & 10179 & 3803 \\
\hline
\end{tabular}

Table 3. Total Processing Time of DJSA Algorithm

\begin{tabular}{|c|c|c|c|c|}
\hline $\begin{array}{c}\text { No. of } \\
\text { Jobs }\end{array}$ & DCLJSA & DMLJSA & DHJSA & DDJSA \\
\hline 50 & 222 & 140 & 174 & 120 \\
\hline 100 & 532 & 262 & 352 & 209 \\
\hline 150 & 473 & 290 & 307 & 232 \\
\hline 200 & 827 & 356 & 451 & 232 \\
\hline 250 & 793 & 431 & 524 & 298 \\
\hline 300 & 899 & 433 & 540 & 255 \\
\hline 350 & 1328 & 564 & 973 & 424 \\
\hline 400 & 2028 & 754 & 1354 & 518 \\
\hline 450 & 679 & 546 & 462 & 390 \\
\hline 500 & 1336 & 677 & 939 & 475 \\
\hline
\end{tabular}

Table 4. Total Processing Cost of W DJSA Algorithm

\begin{tabular}{|c|c|c|c|c|}
\hline $\begin{array}{c}\text { No. of } \\
\text { Jobs }\end{array}$ & WDMLJSA & WDCLJSA & WDDJSA & $\begin{array}{c}\text { WDHJS } \\
\text { A }\end{array}$ \\
\hline 50 & 10732 & 7676 & 5534 & 4036 \\
\hline 100 & 30390 & 28942 & 23745 & 22855 \\
\hline 150 & 49015 & 46261 & 29796 & 28363 \\
\hline 200 & 70534 & 55112 & 35635 & 27608 \\
\hline 250 & 91251 & 58829 & 59209 & 38084 \\
\hline 300 & 89777 & 69827 & 44456 & 34643 \\
\hline 350 & 125437 & 104897 & 83984 & 70545 \\
\hline 400 & 186485 & 163821 & 114402 & 99863 \\
\hline 450 & 124744 & 111332 & 77631 & 68897 \\
\hline 500 & 177196 & 148306 & 117963 & 99139 \\
\hline
\end{tabular}

Table 4. Total Processing Cost of DJSA Algorithm

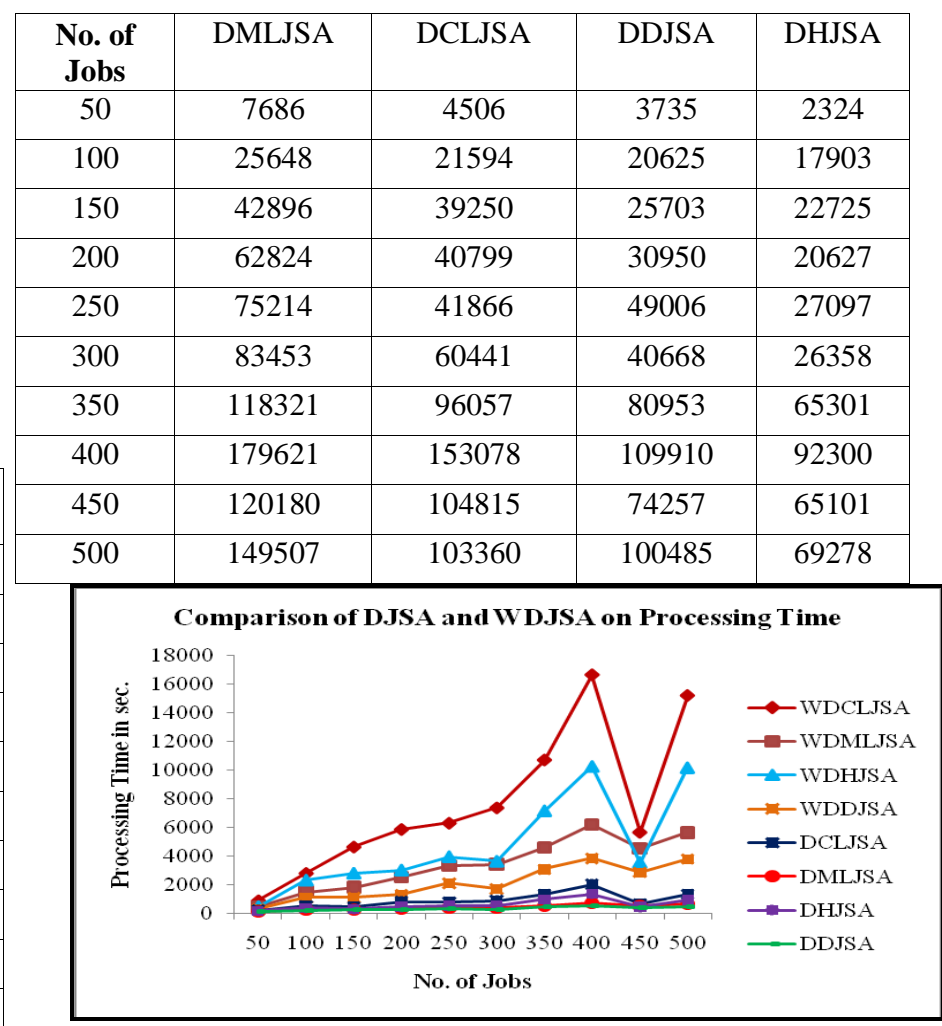

Fig 1: Impact of DJSA and WDJSA on Processing Time

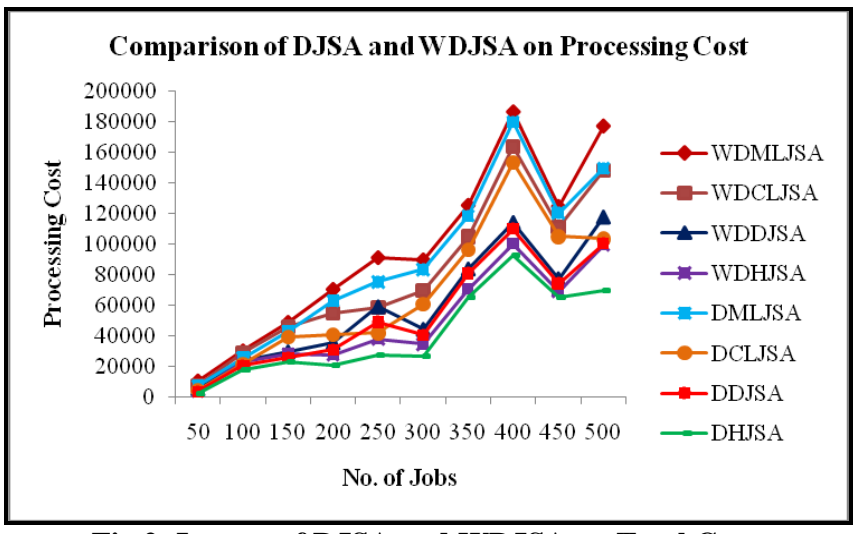

Fig 2: Impact of DJSA and WDJSA on Total Cost

\section{CONCLUSION}

In this paper, we present an effective decentralized job scheduling algorithm for grid environment. The result shows that this algorithm reduces the total processing time and the total cost and finally, the resource utilization is more and the load is balanced across the grid environment. As to future work, is to implement our DJSA algorithm in an actual grid environment. 


\section{REFERENCES}

[1] Syed Nasir Mehmood Shah, Ahmad Kamil Bin Mahmood, and Alan Oxley 2010, "Hybrid Resource Allocation for Grid Computing", in Proceedings of the IEEE Second International Conference on Computer Research and Development, 426 - 431.

[2] D.Yu, T.G.Robertazzi 2003, "Divisible Load Scheduling for Grid Computing", in Proceedings of the International Conference on Parallel and Distributed Computing Systems.

[3] Murugesan, and C.Chellappan 2009, "An Economical Model for Optimal Distribution of Loads for Grid Applications", in Internal Joural of Computer and Network Security, Vol.1, No.1.

[4] P.K.Suri, and Manpreet Singh 2010, "An Efficient Decentralized Load Balancing Algorithm for Grid", in Proceeding ofStar the IEEE Second International Advance Computing Conference, 10 - 13.

[5] M.Balajee, B.Suresh, M.Suneetha, V.Vasudha Rani, and G.Veerraju 2010,'Preemptive Job Scheduling with Priorities and Starvation cum Congestion Avoidance in Clusters ", in Proceedings of the IEEE Second International Conference on Machine Learning and Computing, 225 - 229.

[6] N.Malarvizhi, and Dr.V.Rhymend Uthaiaraj 2009, "A Minimum Time to Release Job Scheduling Algorithm in Computational Grid Environment", in Proceedings of the $5^{\text {th }}$ IEEE Joint International Conference on INC, IMS, and IDC, $13-18$.

[7] I.Foster, and C.Kesselman 1999,"'The Grid: Blueprint for a Future Computing Infrastructure", Morgan Kaufmann Publishers,USA.

[8] M.Baker, R.Buyya, and D.Lafornza 2002,"Grids and Grid Technologies for Wide-area Distributed Computing",
Software-Practice and Experience, Vol.32, No.15, 1437 $-1466$.

[9] I.Foster, C.Kesselman, and S.Tuecke 2001,"The Anatomy of the Grid: Enabling Scalable Virtual Organizations", International Journal on High Performance Computing Applications, Vol.15, No.3, $200-222$.

[10] G.Manimaran, and C.Siva Ram Murthy 1998,"An Efficient Dynamic Scheduling Algorithm for Multiprocessor Real-time Systems", IEEE Transactions on Parallel and Distributed Systems, Vol.9, No.3, 312 319

[11] N.Amano, J.O.Gama, and F.Silva 2003, "Exploiting Parallelism in Decision Tree Induction', in Proceedings from the ECML/PKDD Workshop on Parallel and Distributed Computing for Machine Learning, 13 - 22.

[12] V.Bharadwaj, D.Ghose, and T.G.Robertazzi 2008,"Divisible Load Theory: A New Paradigm for Load Scheduling in Distributed Systems", Cluster Computing, Vol.6, No.1, 7 - 17.

[13] M.Chetper, F.Claeys, B.Dhoedt, F.De Turck, P.Vanrollegham, and P.Demeester 2006,"Dynamic Scheduling of Computationally Intensive Applications on Unreliable Infrastructures", in Proceedings of the Second European Modeling and Simulation Symp.

[14] C.Grimme, J.Lepping, A.Papaspyrou, P.Wieder, R.Yahyapour, A.Oleksiak, O.Waldrich, and W.Ziegler 2007, "Towards a Standards-based Grid Scheduling Architecture", CoreGRID Technical Report TR-0123, Institute on Resource Management and Scheduling.

[15] K.Kurowski, J.Nabrzski, A.Oleksiak, and J.Weglarz 2006, "Scheduling Jobs on the Grid-Multicriteria Approach", Computational Methods in Science and Technology, Vol.12, No.2, 123 - 138 